Int. J. Contemp. Math. Sciences, Vol. 2, 2007, no. 24, 1167 - 1174

\title{
A Subclass of Harmonic Meromorphic Functions
}

\author{
Aini Janteng \\ School of Science and Technology \\ Universiti Malaysia Sabah \\ Locked Bag No. 2073 \\ 88999 Kota Kinabalu, Sabah, Malaysia \\ aini_jg@ums.edu.my \\ Suzeini Abdul Halim \\ Institute of Mathematical Sciences \\ Universiti Malaya \\ 50603 Kuala Lumpur, Malaysia \\ suzeini@um.edu.my
}

\begin{abstract}
Complex-valued harmonic meromorphic functions that are univalent and orientation preserving outside the unit circle $\widetilde{U}$ can be written in the form $f=h+\bar{g}$, where $h$ and $g$ are analytic in $\widetilde{U}$. We define and investigate a subclass of harmonic meromorphic functions. We obtain coefficient conditions, extreme points, distortion bounds, convolution conditions and convex combinations for the above subclass of harmonic meromorphic functions.
\end{abstract}

Keywords: harmonic, meromorphic, univalent

\section{Introduction}

A continuous function $f=u+i v$ is a complex valued harmonic function in a complex domain $\mathcal{D} \subseteq \mathcal{C}$ if both $u$ and $v$ are real harmonic in $\mathcal{D}$. Hengartner and Schober [1], among other things, investigated the family $\sum_{\mathcal{H}}$ of functions $f=$ $h+\bar{g}$ which are harmonic, meromorphic, orientation preserving and univalent in $\widetilde{U}=\{z:|z|>1\}$ where

$$
h(z)=z+\sum_{n=1}^{\infty} a_{n} z^{-n}, \quad g(z)=\sum_{n=1}^{\infty} b_{n} z^{-n}, \quad a_{n} \geq 0, b_{n} \geq 0, z \in \widetilde{U} .
$$


Motivated by the results of [1], Jahangiri and Silverman [3] and Jahangiri [2] studied the classes of functions in $\sum_{\mathcal{H}}$ which are starlike or convex in $\widetilde{U}$. Also let $\sum_{\overline{\mathcal{H}}}$ consisting of functions $f=h+\bar{g}$ where $h$ and $g$ are of the form

$$
h(z)=z+\sum_{n=1}^{\infty}\left|a_{n}\right| z^{-n}, \quad g(z)=-\sum_{n=1}^{\infty}\left|b_{n}\right| z^{-n}, \quad z \in \widetilde{U} .
$$

Now, we consider the class of functions as follows:

Definition 1.1 Let $f \in \sum_{\mathcal{H}}$. Then $f \in \sum_{\mathcal{H}} \mathcal{S}^{\star}(\lambda, \alpha)$, if and only if, for $0 \leq \lambda \leq 1$ and $0 \leq \alpha<1$,

$$
\operatorname{Re}\left\{\frac{z h^{\prime}(z)-\overline{z g^{\prime}(z)}}{\lambda\left(z h^{\prime}(z)-\overline{z g^{\prime}(z)}\right)+(1-\lambda)(h(z)+\overline{g(z)})}\right\} \geq \alpha .
$$

Also, we let $\sum_{\overline{\mathcal{H}}} \mathcal{S}^{\star}(\lambda, \alpha)=\sum_{\mathcal{H}} \mathcal{S}^{\star}(\lambda, \alpha) \cap \sum_{\overline{\mathcal{H}}}$.

The following theorem proved by Jahangiri and Silverman in [3] will be used throughout in this paper.

Theorem 1.1 ([3]) Let $f=h+\bar{g}$ with $h$ and $g$ of the form (1). If

$$
\sum_{n=1}^{\infty} n\left|a_{n}\right|+\sum_{n=1}^{\infty} n\left|b_{n}\right| \leq 1
$$

then $f$ is harmonic, orientation preserving, univalent in $\widetilde{U}$ and $f \in \sum_{\mathcal{H}} \mathcal{S}^{\star}(\lambda, \alpha)$.

\section{Results}

We begin the results with a sufficient coefficient condition for functions in $\sum_{\mathcal{H}} \mathcal{S}^{\star}(\lambda, \alpha)$.

Theorem 2.1 Let $f=h+\bar{g}$ be of the form (1). If

$$
\sum_{n=1}^{\infty} \frac{n+\alpha-\alpha \lambda(n+1)}{1-\alpha}\left|a_{n}\right|+\sum_{n=1}^{\infty} \frac{n-\alpha-\alpha \lambda(n-1)}{1-\alpha}\left|b_{n}\right| \leq 1
$$

then $f$ is harmonic, orientation preserving, univalent in $\widetilde{U}$ and $f \in \sum_{\mathcal{H}} \mathcal{S}^{\star}(\lambda, \alpha)$. Proof. Since $n \leq \frac{n+\alpha-\alpha \lambda(n+1)}{1-\alpha}$ and $n \leq \frac{n-\alpha-\alpha \lambda(n-1)}{1-\alpha}$, it follows from Theorem 1.1 that $f$ is harmonic, orientation preserving and univalent in $\widetilde{U}$. Now, we only need to show that if (5) holds then

$$
\operatorname{Re}\left\{\frac{z h^{\prime}(z)-\overline{z g^{\prime}(z)}}{\lambda\left(z h^{\prime}(z)-\overline{z g^{\prime}(z)}\right)+(1-\lambda)(h(z)+\overline{g(z)})}\right\}=\operatorname{Re} \frac{A(z)}{B(z)} \geq \alpha .
$$


Using the fact that $\operatorname{Re}(w) \geq \alpha$ if and only if $|1-\alpha+w| \geq|1+\alpha-w|$, it sufficies to show that

$$
|A(z)+(1-\alpha) B(z)|-|A(z)-(1+\alpha) B(z)| \geq 0
$$

where

$$
A(z)=z h^{\prime}(z)-\overline{z g^{\prime}(z)}
$$

and

$$
B(z)=\lambda\left(z h^{\prime}(z)-\overline{z g^{\prime}(z)}\right)+(1-\lambda)(h(z)+\overline{g(z)}) .
$$

Substituting for $A(z)$ and $B(z)$ in $(6)$, we obtain

$$
\begin{aligned}
& |A(z)+(1-\alpha) B(z)|-|A(z)-(1+\alpha) B(z)| \\
& =\mid(2-\alpha) z-\sum_{n=1}^{\infty}[n+\alpha-\alpha \lambda(n+1)+\lambda n+\lambda-1] a_{n} z^{-n} \\
& +\sum_{n=1}^{\infty}[n-\alpha-\alpha \lambda(n-1)+\lambda n-\lambda+1] b_{n} \bar{z}^{-n} \mid \\
& -\mid-\alpha z-\sum_{n=1}^{\infty}[n+\alpha-\alpha \lambda(n+1)-\lambda n-\lambda+1] a_{n} z^{-n} \\
& +\sum_{n=1}^{\infty}[n-\alpha-\alpha \lambda(n-1)-\lambda n+\lambda-1] b_{n} \bar{z}^{-n} \mid \\
& \geq(2-\alpha)|z|-\sum_{n=1}^{\infty}[n+\alpha-\alpha \lambda(n+1)+\lambda n+\lambda-1]\left|a_{n}\right||z|^{-n} \\
& -\sum_{n=1}^{\infty}[n-\alpha-\alpha \lambda(n-1)+\lambda n-\lambda+1]\left|b_{n}\right||z|^{-n}-\alpha|z| \\
& -\sum_{n=1}^{\infty}[n+\alpha-\alpha \lambda(n+1)-\lambda n-\lambda+1]\left|a_{n}\right||z|^{-n} \\
& -\sum_{n=1}^{\infty}[n-\alpha-\alpha \lambda(n-1)-\lambda n+\lambda-1]\left|b_{n}\right||z|^{-n} \\
& \geq 2(1-\alpha)|z|\left\{1-\sum_{n=1}^{\infty} \frac{[n+\alpha-\alpha \lambda(n+1)]}{1-\alpha}\left|a_{n}\right|-\sum_{n=1}^{\infty} \frac{[n-\alpha-\alpha \lambda(n-1)]}{1-\alpha}\left|b_{n}\right|\right\} \\
& \geq 0, \quad b y(5) \text {. }
\end{aligned}
$$

Next we show that the bound (5) is also necessary for functions in $\sum_{\overline{\mathcal{H}}} \mathcal{S}^{\star}(\lambda, \alpha)$. 
Theorem 2.2 Let $f=h+\bar{g}$ with $h$ and $g$ of the form (2). Then $f \in$ $\sum_{\overline{\mathcal{H}}} \mathcal{S}^{\star}(\lambda, \alpha)$ if and only if

$$
\sum_{n=1}^{\infty} \frac{n+\alpha-\alpha \lambda(n+1)}{1-\alpha}\left|a_{n}\right|+\sum_{n=1}^{\infty} \frac{n-\alpha-\alpha \lambda(n-1)}{1-\alpha}\left|b_{n}\right| \leq 1
$$

Proof. In view of Theorem 2.1, we only need to show that $f$ is not in $\sum_{\overline{\mathcal{H}}} \mathcal{S}^{\star}(\lambda, \alpha)$ if condition (7) does not hold. We note that a necessary and sufficient condition for $f=h+\bar{g}$ given by (2) to be in $\sum_{\mathcal{H}} \mathcal{S}^{\star}(\lambda, \alpha)$ is that the coefficient condition (3) to be satisfied. Equivalently, we must have

$$
\begin{aligned}
& \operatorname{Re}\left\{\frac{z h^{\prime}(z)-\overline{z g^{\prime}(z)}}{\lambda\left(z h^{\prime}(z)-\overline{z g^{\prime}(z)}\right)+(1-\lambda)(h(z)+\overline{g(z)})}-\alpha\right\} \\
& =\operatorname{Re}\left\{\frac{(1-\alpha) z-\sum_{n=1}^{\infty}[n+\alpha-\alpha \lambda(n+1)]\left|a_{n}\right| z^{-n}-\sum_{n=1}^{\infty}[n-\alpha-\alpha \lambda(n-1)]\left|b_{n}\right| \bar{z}-n}{z+\sum_{n=1}^{\infty}[1-\lambda n-\lambda]\left|a_{n}\right| z^{-n}-\sum_{n=1}^{\infty}[\lambda n-\lambda+1]\left|b_{n}\right| \bar{z}-n}\right\} \\
& \geq 0 .
\end{aligned}
$$

This inequality must hold for all $z \in \widetilde{U}$. Letting $z=r>1$, we need

$\operatorname{Re}\left\{\frac{1-\alpha-\sum_{n=1}^{\infty}[n+\alpha-\alpha \lambda(n+1)]\left|a_{n}\right| r^{-n-1}-\sum_{n=1}^{\infty}[n-\alpha-\alpha \lambda(n-1)]\left|b_{n}\right| r^{-n-1}}{1+\sum_{n=1}^{\infty}[1-\lambda n-\lambda]\left|a_{n}\right| r^{-n-1}-\sum_{n=1}^{\infty}[\lambda n-\lambda+1]\left|b_{n}\right| r^{-n-1}}\right\}=\frac{A(r)}{B(r)} \geq 0$.

If condition (7) does not hold then $A(r)$ is negative for $r$ sufficiently close to 1 . Thus there exists $z_{0}=r_{0}>1$ for which the quotient $\frac{A(r)}{B(r)}$ is negative. This contradicts the required condition that $\frac{A(r)}{B(r)} \geq 0$ and so the proof is complete.

The growth result for functions in $\sum_{\overline{\mathcal{H}}} \mathcal{S}^{\star}(\lambda, \alpha)$ is discussed in the following theorem.

Theorem 2.3 If $f \in \sum_{\overline{\mathcal{H}}} \mathcal{S}^{\star}(\lambda, \alpha)$ then

$$
|f(z)| \leq r+(1-\alpha) r^{-1}, \quad|z|=r>1
$$

and

$$
|f(z)| \geq r-(1-\alpha) r^{-1}, \quad|z|=r>1 .
$$

Proof. Let $f \in \sum_{\overline{\mathcal{H}}} \mathcal{S}^{\star}(\lambda, \alpha)$. Taking the absolute value of $f$ we have

$$
\begin{aligned}
|f(z)| & =\left|z+\sum_{n=1}^{\infty}\right| a_{n}\left|z^{-n}-\sum_{n=1}^{\infty}\right| b_{n}\left|\bar{z}^{-n}\right| \\
& \leq r+\sum_{n=1}^{\infty}\left(\left|a_{n}\right|+\left|b_{n}\right|\right) r^{-n} \\
& \leq r+\sum_{n=1}^{\infty}\left(\left|a_{n}\right|+\left|b_{n}\right|\right) r^{-1}
\end{aligned}
$$


Harmonic meromorphic functions

$$
\begin{aligned}
& \leq r+\sum_{n=1}^{\infty}\left[(n+\alpha-\alpha \lambda(n+1))\left|a_{n}\right|+(n-\alpha-\alpha \lambda(n-1))\left|b_{n}\right|\right] r^{-1} \\
& \leq r+(1-\alpha) r^{-1}
\end{aligned}
$$

and

$$
\begin{aligned}
|f(z)| & =\left|z+\sum_{n=1}^{\infty}\right| a_{n}\left|z^{-n}-\sum_{n=1}^{\infty}\right| b_{n}\left|\bar{z}^{-n}\right| \\
& \geq r-\sum_{n=1}^{\infty}\left(\left|a_{n}\right|+\left|b_{n}\right|\right) r^{-n} \\
& \geq r-\sum_{n=1}^{\infty}\left(\left|a_{n}\right|+\left|b_{n}\right|\right) r^{-1} \\
& \geq r-\sum_{n=1}^{\infty}\left[(n+\alpha-\alpha \lambda(n+1))\left|a_{n}\right|+(n-\alpha-\alpha \lambda(n-1))\left|b_{n}\right|\right] r^{-1} \\
& \geq r-(1-\alpha) r^{-1} . \square
\end{aligned}
$$

Next, we determine the extreme points of closed hulls of $\sum_{\overline{\mathcal{H}}} \mathcal{S}^{\star}(\lambda, \alpha)$ denoted by $\operatorname{clco} \sum_{\overline{\mathcal{H}}} \mathcal{S}^{\star}(\lambda, \alpha)$.

Theorem $2.4 f \in \operatorname{clco} \sum_{\overline{\mathcal{H}}} \mathcal{S}^{\star}(\lambda, \alpha)$ if and only if $f(z)=\sum_{n=0}^{\infty}\left(X_{n} h_{n}+Y_{n} g_{n}\right)$ where

$$
\begin{aligned}
& h_{0}(z)=z, h_{n}(z)=z+\frac{1-\alpha}{n+\alpha-\alpha \lambda(n+1)} z^{-n}(n=1,2,3, \ldots), \\
& g_{0}(z)=z, g_{n}(z)=z-\frac{1-\alpha}{n-\alpha-\alpha \lambda(n-1)} \bar{z}^{-n}(n=1,2,3, \ldots),
\end{aligned}
$$

$\sum_{n=0}^{\infty}\left(X_{n}+Y_{n}\right)=1, X_{n} \geq 0$ and $Y_{n} \geq 0$.

Proof. For $h_{n}$ and $g_{n}$ as given above, we may write

$$
\begin{aligned}
f(z)= & \sum_{n=0}^{\infty}\left(X_{n} h_{n}+Y_{n} g_{n}\right) \\
= & X_{0} h_{0}+Y_{0} g_{0} \\
& +\sum_{n=1}^{\infty}\left[X_{n}\left(z+\frac{1-\alpha}{n+\alpha-\alpha \lambda(n+1)} z^{-n}\right)+Y_{n}\left(z-\frac{1-\alpha}{n-\alpha-\alpha \lambda(n-1)} \bar{z}^{-n}\right)\right] \\
= & \sum_{n=0}^{\infty}\left(X_{n}+Y_{n}\right) z+\sum_{n=1}^{\infty} \frac{1-\alpha}{n+\alpha-\alpha \lambda(n+1)} X_{n} z^{-n}-\sum_{n=1}^{\infty} \frac{1-\alpha}{n-\alpha-\alpha \lambda(n-1)} Y_{n} \bar{z}^{-n} .
\end{aligned}
$$

Then

$$
\sum_{n=1}^{\infty}(n+\alpha-\alpha \lambda(n+1))\left(\frac{1-\alpha}{n+\alpha-\alpha \lambda(n+1)} X_{n}\right)
$$




$$
\begin{aligned}
+\sum_{n=1}^{\infty}(n-\alpha-\alpha \lambda(n-1))\left(\frac{1-\alpha}{n-\alpha-\alpha \lambda(n-1)} Y_{n}\right) \\
=(1-\alpha) \sum_{n=1}^{\infty}\left(X_{n}+Y_{n}\right) \\
=(1-\alpha)\left[1-\left(X_{0}+Y_{0}\right)\right] \\
\leq 1-\alpha .
\end{aligned}
$$

Therefore $f \in \operatorname{clco} \sum_{\overline{\mathcal{H}}} \mathcal{S}^{\star}(\lambda, \alpha)$.

Conversely, suppose that $f \in \operatorname{clco} \sum_{\overline{\mathcal{H}}} \mathcal{S}^{\star}(\lambda, \alpha)$. Then we write

$$
f(z)=z+\sum_{n=1}^{\infty}\left|a_{n}\right| z^{-n}-\sum_{n=1}^{\infty}\left|b_{n}\right| \bar{z}^{-n}
$$

and

$$
\sum_{n=1}^{\infty}\left[\frac{n+\alpha-\alpha \lambda(n+1)}{1-\alpha}\left|a_{n}\right|+\frac{n-\alpha-\alpha \lambda(n-1)}{1-\alpha}\left|b_{n}\right|\right] \leq 1 .
$$

Setting

$$
X_{n}=\frac{n+\alpha-\alpha \lambda(n+1)}{1-\alpha}\left|a_{n}\right|,(n=1,2,3, \ldots)
$$

and

$$
Y_{n}=\frac{n-\alpha-\alpha \lambda(n-1)}{1-\alpha}\left|b_{n}\right|,(n=1,2,3, \ldots),
$$

where $0 \leq X_{0} \leq 1$ and $Y_{0}=1-X_{0}-\sum_{n=1}^{\infty}\left(X_{n}+Y_{n}\right)$, we get $f(z)=$ $\sum_{n=0}^{\infty}\left(X_{n} h_{n}+Y_{n} g_{n}\right)$ as required.

For harmonic functions $f(z)=z+\sum_{n=1}^{\infty}\left|a_{n}\right| z^{-n}-\sum_{n=1}^{\infty}\left|b_{n}\right| \bar{z}^{-n}$ and $F(z)=$ $z+\sum_{n=1}^{\infty}\left|A_{n}\right| z^{-n}-\sum_{n=1}^{\infty}\left|B_{n}\right| \bar{z}^{-n}$, we define the convolution of $f$ and $F$ as

$$
(f \star F)(z)=z+\sum_{n=1}^{\infty}\left|a_{n} A_{n}\right| z^{-n}-\sum_{n=1}^{\infty}\left|b_{n} B_{n}\right| \bar{z}^{-n} .
$$

In the next theorem, we examine the convolution properties of the class $\sum_{\overline{\mathcal{H}}} \mathcal{S}^{\star}(\lambda, \alpha)$.

Theorem 2.5 For $0 \leq \beta \leq \alpha<1$, let $f \in \sum_{\overline{\mathcal{H}}} \mathcal{S}^{\star}(\lambda, \alpha)$ and $F \in \sum_{\overline{\mathcal{H}}} \mathcal{S}^{\star}(\lambda, \alpha)$. Then $(f \star F) \in \sum_{\overline{\mathcal{H}}} \mathcal{S}^{\star}(\lambda, \alpha) \subset \sum_{\overline{\mathcal{H}}} \mathcal{S}^{\star}(\lambda, \alpha)$.

Proof. Write $f(z)=z+\sum_{n=1}^{\infty}\left|a_{n}\right| z^{-n}-\sum_{n=1}^{\infty}\left|b_{n}\right| \bar{z}^{-n}$ and $F(z)=z+$ $\sum_{n=1}^{\infty}\left|A_{n}\right| z^{-n}-\sum_{n=1}^{\infty}\left|B_{n}\right| \bar{z}^{-n}$. Then the convolution of $f$ and $F$ is given by $(8)$. 
Note that $\left|A_{n}\right| \leq 1$ and $\left|B_{n}\right| \leq 1$ since $F \in \sum_{\overline{\mathcal{H}}} \mathcal{S}^{\star}(\lambda, \alpha)$. Then we have

$$
\begin{aligned}
& \sum_{n=1}^{\infty}\left[[n+\alpha-\alpha \lambda(n+1)]\left|a_{n}\right|\left|A_{n}\right|+[n-\alpha-\alpha \lambda(n-1)]\left|b_{n}\right|\left|B_{n}\right|\right] \\
& \leq \sum_{n=1}^{\infty}\left[[n+\alpha-\alpha \lambda(n+1)]\left|a_{n}\right|+[n-\alpha-\alpha \lambda(n-1)]\left|b_{n}\right|\right] .
\end{aligned}
$$

Therefore, $(f \star F) \in \sum_{\overline{\mathcal{H}}} \mathcal{S}^{\star}(\lambda, \alpha) \subset \sum_{\overline{\mathcal{H}}} \mathcal{S}^{\star}(\lambda, \alpha)$ since the right hand side of the above inequality is bounded by $1-\alpha$ while $1-\alpha \leq 1-\beta$.

Now, we determine the convex combination properties of the members of $\sum_{\overline{\mathcal{H}}} \mathcal{S}^{\star}(\lambda, \alpha)$.

Theorem 2.6 The class $\sum_{\overline{\mathcal{H}}} \mathcal{S}^{\star}(\lambda, \alpha)$ is closed under convex combination.

Proof. For $i=1,2,3, \ldots$, suppose that $f_{i} \in \sum_{\overline{\mathcal{H}}} \mathcal{S}^{\star}(\lambda, \alpha)$ where $f_{i}$ is given by

$$
f_{i}(z)=z+\sum_{n=1}^{\infty}\left|a_{n, i}\right| z^{-n}-\sum_{n=1}^{\infty}\left|b_{n, i}\right| \bar{z}^{-n} .
$$

For $\sum_{i=1}^{\infty} c_{i}=1,0 \leq c_{i} \leq 1$, the convex combinations of $f_{i}$ may be written as

$$
\begin{aligned}
\sum_{i=1}^{\infty} c_{i} f_{i}(z) & =c_{1} z+\sum_{n=1}^{\infty} c_{1}\left|a_{n, 1}\right| z^{-n}-\sum_{n=1}^{\infty} c_{1}\left|b_{n, 1}\right| \bar{z}^{-n}+c_{2} z+\sum_{n=1}^{\infty} c_{2}\left|a_{n, 2}\right| z^{-n}-\sum_{n=1}^{\infty} c_{2}\left|b_{n, 2}\right| \bar{z}^{-n} \ldots \\
& =z \sum_{i=1}^{\infty} c_{i}+\sum_{n=1}^{\infty}\left(\sum_{i=1}^{\infty} c_{i}\left|a_{n, i}\right|\right) z^{-n}-\sum_{n=1}^{\infty}\left(\sum_{i=1}^{\infty} c_{i}\left|b_{n, i}\right|\right) \bar{z}^{-n} \\
& =z+\sum_{n=1}^{\infty}\left(\sum_{i=1}^{\infty} c_{i}\left|a_{n, i}\right|\right) z^{-n}-\sum_{n=1}^{\infty}\left(\sum_{i=1}^{\infty} c_{i}\left|b_{n, i}\right|\right) \bar{z}^{-n}
\end{aligned}
$$

Next, consider

$$
\begin{aligned}
\sum_{n=1}^{\infty}( & {\left.[n+\alpha-\alpha \lambda(n+1)]\left|\sum_{i=1}^{\infty} c_{i}\right| a_{n, i} \mid\right)+\sum_{n=1}^{\infty}\left([n-\alpha-\alpha \lambda(n-1)]\left|\sum_{i=1}^{\infty} c_{i}\right| b_{n, i} \mid\right) } \\
= & c_{1} \sum_{n=1}^{\infty}[n+\alpha-\alpha \lambda(n+1)]\left|a_{n, 1}\right|+\ldots+c_{m} \sum_{n=1}^{\infty}[n+\alpha-\alpha \lambda(n+1)]\left|a_{n, m}\right|+\ldots \\
& +c_{1} \sum_{n=1}^{\infty}[n-\alpha-\alpha \lambda(n-1)]\left|b_{n, 1}\right|+\ldots+c_{m} \sum_{n=1}^{\infty}[n-\alpha-\alpha \lambda(n-1)]\left|b_{n, m}\right|+\ldots \\
= & \sum_{i=1}^{\infty} c_{i}\left\{\sum_{n=1}^{\infty}[n+\alpha-\alpha \lambda(n+1)]\left|a_{n, i}\right|+\sum_{n=1}^{\infty}[n-\alpha-\alpha \lambda(n-1)]\left|b_{n, i}\right|\right\} .
\end{aligned}
$$


Now, $f_{i} \in \sum_{\overline{\mathcal{H}}} \mathcal{S}^{\star}(\lambda, \alpha)$, therefore from Theorem 2.2, we have

$$
\sum_{n=1}^{\infty}[n+\alpha-\alpha \lambda(n+1)]\left|a_{n, i}\right|+\sum_{n=1}^{\infty}[n-\alpha-\alpha \lambda(n-1)]\left|b_{n, i}\right| \leq 1-\alpha .
$$

Hence

$$
\begin{aligned}
& \sum_{n=1}^{\infty}\left([n+\alpha-\alpha \lambda(n+1)]\left|\sum_{i=1}^{\infty} c_{i}\right| a_{n, i}||\right)+\sum_{n=1}^{\infty}\left([n-\alpha-\alpha \lambda(n-1)]\left|\sum_{i=1}^{\infty} c_{i}\right| b_{n, i}||\right) \\
& \leq(1-\alpha) \sum_{i=1}^{\infty} c_{i} \\
& =1-\alpha .
\end{aligned}
$$

By using Theorem 2.2 again, we have $\sum_{i=1}^{\infty} c_{i} f_{i} \in \sum_{\overline{\mathcal{H}}} \mathcal{S}^{\star}(\lambda, \alpha)$.

\section{References}

[1] W. Hengartner and G. Schober, Univalent harmonic functions, Trans. Amer. Math. Soc., 299 (1987), 1-31.

[2] J.M. Jahangiri, Harmonic meromorphic starlike functions, Bull. Korean Math. Soc., 37 (2000), 291-301.

[3] J.M. Jahangiri and H. Silverman, Meromorphic univalent harmonic functions with negative coefficients, Bull. Korean Math. Soc., 36 (1999), 763770 .

Received: April 23, 2007 